\title{
Radiographic and Clinical Outcomes of Dynesys Dynamic Stabilization Versus Instrumented Fusion for Degenerative Lumbar Spine Diseases With a Minimum Follow-Up Period of 2 Years: A Meta-Analysis
}

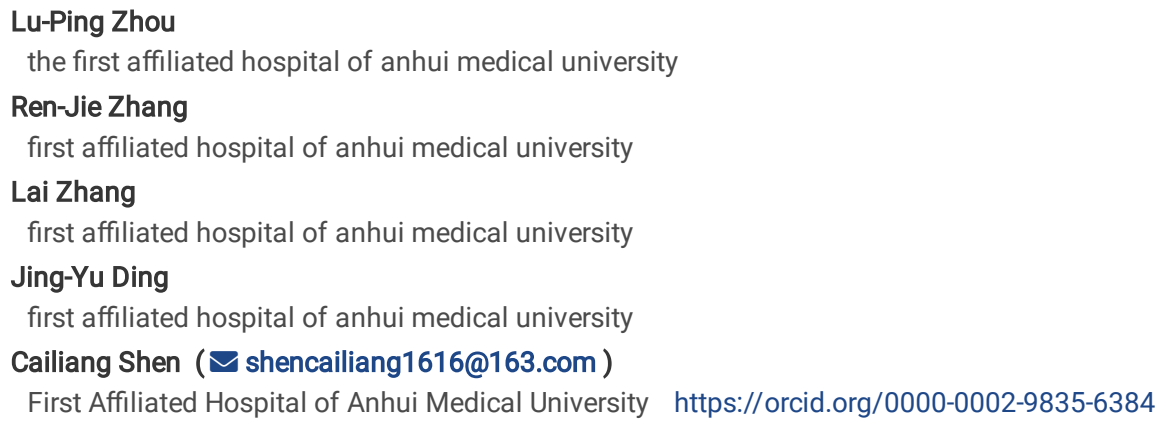




\section{Abstract}

Objective

Dynesys stabilization (DS) is utilized to preserve mobility at the instrumental segments and prevent adjacent segment pathology (ASP) in clinical practice. However, the advantages of DS method remain controversial. This meta-analysis was to compare the radiographic and clinical outcomes between DS and instrumented fusion in patients with or without grade I spondylolisthesis at a minimum follow-up period of 2 years.

Methods

We conducted a comprehensive search of PubMed, EMBASE, Cochrane, and Web of Science databases for potentially eligible articles. Clinical outcomes were assessed in terms of surgical time, intraoperative blood loss, length of hospital stay, in-hospital complications, VAS and ODI scores, screw loosening and breakage, and reoperation. Radiographic outcomes were assessed in terms of postoperative range of movement (ROM) and disc heigh. Moreover, adjacent segment degeneration (ASDeg) and adjacent segment disease (ASDis) were evaluated. Data are expressed as risk ratio or standardized mean difference with the associated 95\% confidence intervals. The meta-analysis was performed using RevMan 5.3 and STATA 15.1 software.

Results

Fourteen studies with 1078 patients were included in the meta-analysis. The mean durations of follow-up ranged from $26.64 \pm 5.16$ months to $93.6 \pm 16.5$ months. The DS group was associated with significantly shorter surgical time, fewer intraoperative blood loss, shorter length of hospital stay, less in-hospital complications, lower postoperative VAS scores for low-back and leg pain than the fusion group $(P<0.05)$. Moreover, the ROM at the stabilized segments of the fusion group decreased significantly $(P<0.01)$ and that at the adjacent segments increased significantly compared with those of the Dynesys group $(P<$ 0.01). Additionally, the Dynesys group showed significantly less ASDeg than the fusion group $(P<0.01)$ but showed no significant advantage over the fusion group in terms of preventing ASDis $(P=0.33)$. Furthermore, the Dynesys group was insignificantly different from the fusion group in terms of ODI, screw loosening, screw breakage, surgical reoperation, and disc heigh at stabilized and adjacent segments $(P>0.05)$.

Conclusion

Dynesys stabilization showed satisfactory clinical outcomes and provided additional benefits in preserving the motion at the stabilized segments and limiting the hypermobility at the adjacent segments compared with fusion method. Dynesys stabilization represented a complementary choice in the treatment of degenerative spinal lumbar disease with or without grade I spondylolisthesis.

\section{Introduction}

Lumbar fusion has been considered as the gold standard and is widely adapted worldwide for treatment of lumbar degenerative pathologies over the last decades. ${ }^{1,2}$ However, instrumented fusion causes complications, including instrumentation failure, pseudoarthrosis, and pain in donor areas. ${ }^{3-5}$ Moreover, the preservation of lumbar motion is less considered in fusion instrument, leading to the limited motility of stabilized segments and the increased load on adjacent segments, which may increase the risk of adjacent segment degeneration (ASDeg), adjacent segment disease (ASDis), and severe postoperative functional disabilities. ${ }^{6-8}$

In recent years, non-fusion systems have been applied clinically to prevent the incidence of adjacent segment pathology (ASP, including ASDeg and ASDis) after lumbar surgeries. ${ }^{9,} 10$ The Dynesys posterior dynamic stabilization system (Zimmer Inc., Warsaw, IN, USA), which was first introduced in 1994, is one of the most frequently used dynamic stabilization devices. ${ }^{11}$ This system consists of pedicle screws (Ti alloy), polyethylene-terephtalate (PET) cords, and polycarbonate-urethane (PCU) spacers for stabilization of stabilized segments, restoration of normal segmental kinematics, and preservation of adjacent motion to prevent the instability and decrease the incidence of ASP. ${ }^{5,12-14}$ Many studies reported that the Dynesys system (DS) showed significant improvement in terms of visual analog scale (VAS) pain scores, Oswestry disability index (ODI) scores, trauma severity, and recovery time than fusion method. ${ }^{4,15-19}$ Moreover, the range of movement (ROM) at adjacent segments and along with the load across the intervertebral and adjacent discs was reduced. $4,5,7,12,16$

However, many studies showed contradicting results. In previous works, DS showed no remarkable advantages over fusion method in terms of surgical time,

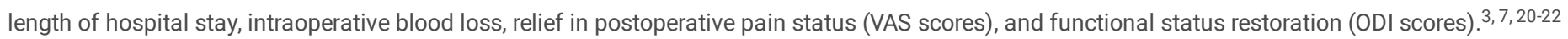
Meanwhile, DS was reported to be associated with high rates of ASDeg and ASDis. $5,6,23$ Although DS is a safe and effective treatment in terms of short-term outcomes, the functional status, preservation of mobility, and prevention of ASP in medium- and long-term periods remain controversial. Some clinical and radiographical measurements showed opposite results in different durations of follow-up. ${ }^{7,16,20,22,24}$ Thus, the purpose of this meta-analysis was to compare the radiographic and clinical outcomes of dynamic DS and instrumented fusion for treatment of degenerative lumbar spine diseases with a minimum followup period of 2 years.

\section{Methods}

\section{Search strategy}

This meta-analysis was performed based on the Preferred Reporting Items for Systematic Reviews and Meta-analyses (PRISMA) guidelines. ${ }^{25}$ A systematic search on PubMed, EMBASE, Cochrane, and Web of Science databases was conducted to identify potentially eligible articles from the inception to May 23 , 
2020, without language restriction. Search was performed using the terms "Dynesys" or "dynamic" or "semi-rigid" and "fusion" and "lumbar." The detailed search strategy is reported in Supplementary Table 1. Two reviewers independently searched all the titles and abstracts. The reference lists of relevant studies on DS were also reviewed for additional literature. Full-text articles were obtained if there were any uncertainties. Any disagreement was settled by a third reviewer.

\section{Inclusion and exclusion criteria}

The inclusion and exclusion of the studies for the meta-analysis were based on the following criteria. (1) For participants, the study population consisted of patients who satisfied the following criteria: aged 18 years or older; mean follow-up period $\geq 2$ years; suffering from lumbar degenerative diseases, including disc herniation, lumbar spinal stenosis, and grade I degenerative spondylolisthesis; and the number of fixed segments ranging from 1 to 4 in the lumbar. Studies on patients with grade II or higher spondylolisthesis, ankylosis spondylitis, spinal tumor, and severe spinal deformity were excluded. (2) For interventions, the intervention in the experimental group was dynamic DS. Studies on hybrid dynamic stabilization and other kinds of dynamic stabilization including Coflex, Wallis, and X-stop systems were excluded. (3) For comparisons, the intervention in the control group was instrumented fusion methods, including posterior lumbar interbody fusion, transforaminal lumbar interbody fusion, and posterolateral fusion. (4) For outcomes, studies were eligible if they satisfied at least one of the following outcomes: clinical outcomes during hospital stay (surgical time, intraoperative blood loss, length of hospital stay, and inhospital complications), clinical outcomes at final follow-up (VAS and ODI scores, screw loosening and breakage, surgical reoperation), radiographical outcomes (postoperative ROM and disc heigh), and ASP (ASDeg and ASDis). The in-hospital complications included nerve root injuries, dural tear, delirium, deep venous thrombosis, incision infection, dyspnea, and incision fat liquefaction. ASDeg (radiographic ASD) represents radiographic etiologies, involving loss of disc height, disc degeneration, stenosis, instability, or hypertrophic facet arthritis, regardless of the presence of symptoms. ${ }^{8}$ ASDis (symptomatic ASD) is defined as clinical symptoms corelated with one of radiographic changes in adjacent segments. ${ }^{2,8}(5)$ For study design, randomized controlled trials (RCTs) or comparative studies were eligible. Case-control studies, case reports, reviews, and conference reports were excluded.

\section{Risk of bias assessment}

Cochrane risk-of-bias criteria ${ }^{26}$ and Newcastle-Ottawa Scale (NOS) ${ }^{27}$ were utilized to assess the methodological quality of the included RCTs and comparative studies, respectively.

The included randomized controlled trials were evaluated based on randomization sequence generation, allocation concealment, blinding of participants and personnel, blinding of outcome assessment, incomplete outcome data, selective reporting, and other bias. We defined other biases as different baseline characteristics in the experimental and control groups. The bias of domains was qualified as low risk, high risk, or unclear risk. Meanwhile, comparative cohort studies were assessed in terms of selection of patients, comparability, and outcomes of the case and control groups, with scores ranging from 0 to 9 . The studies were evaluated as high quality (score 8 or 9), moderate quality (score 6 or 7), and low quality (score 5 or less).

\section{Data extraction}

Two reviewers independently extracted relevant data from the qualified studies. Any disagreement was settled by a third reviewer. Leading author, publication year, study design, country of origin, study period, age, sex distribution, sample size, experimental and control interventions, number of treated segments, fusion type, and follow-up period were extracted from the included studies.

\section{Statistical analysis}

Risk ratio (RR) and its 95\% confidence interval ( $\mathrm{Cl}$ ) were calculated for dichotomous data. Mean difference (MD) and its $95 \% \mathrm{Cl}$ were calculated for continuous data. We used $\mathrm{I}^{2}$ and chi-squared tests at a significance level of $P<0.05$ for assessment of statistical heterogeneity. A fixed-effects model was utilized if no evidence of heterogeneity $\left(1^{2}<50 \%\right)$ was observed among the studies. Otherwise, a random-effects model was used. Subgroup and sensitivity analyses were performed to investigate the source of heterogeneity. In addition, publication bias was evaluated by Egger tests when the number of included studies was 8 or more. Except for publication bias assessed by STATA version 15.1, statistical analysis was performed with Review Manager version 5.3. All tests were twotailed, and $P<0.05$ was considered statistically significant.

\section{Results}

\section{Study search}

The process for literature search is summarized in Figure 1. A total of 2488 potential papers were inspected from the electronic searches, and 909 studies were excluded because of duplication. After assessing the titles and abstracts, 1531 studies were removed and the remaining 48 articles were downloaded for fulltext verification. Finally, one prospective randomized controlled trial ${ }^{21}$, five prospective clinical studies ${ }^{3,16,18,19,28}$, and eight retrospective studies ${ }^{4,7,15,17,20,}$ $22,24,29$ were deemed eligible and included in the meta-analysis.

\section{Main characteristics of the included studies}

Table 1 summarizes the main characteristics of the included studies. The baseline information of the two groups of the 14 studies was balanced and comparable. All of the studies were conducted on a single site. Among the included studies, 10 studies $3,4,16,18-22,24,28$ were conducted in China, and one study was conducted in Germany ${ }^{7}$, France ${ }^{15}$, Italy ${ }^{29}$, and the UK ${ }^{17}$.

\section{Risk of bias in the included studies}


The risk of bias for the included studies are shown in Supplementary Tables $\mathbf{2}$ and $\mathbf{3}$. The only randomized controlled trial ${ }^{21}$ was regarded as high quality and showed adequate randomization and allocation concealment. However, the blinding of participants and personnel was of high risk. For the comparative cohort studies assessed by NOS, six studies $3,7,16,19,24,29$ were considered to be sufficiently high quality, and the seven remaining studies ${ }^{4,15,17,18,20,22,28}$ were evaluated as moderate quality.

\section{Meta-analysis results}

\section{Clinical outcomes during hospital stay}

Surgical time, length of hospital stay, and intraoperative blood loss

Six studies $3,4,19-21,24$, three studies ${ }^{3,4,21}$, and six studies $3,4,19-21,24$ provided data on surgical time, length of hospital stay, and intraoperative blood loss between Dynesys and fusion groups. The combined results revealed that the Dynesys group was associated with significantly shorter surgical time $(\mathrm{MD}=-$ $24.66,95 \% \mathrm{Cl}$ of -35.47 to $-13.85, P<0.01, \mathrm{I}^{2}=88 \%$; Figure 2$)$, shorter length of hospital stay $\left(\mathrm{MD}=-1.84,95 \% \mathrm{Cl}\right.$ of -2.28 to $-1.41, P<0.01, \mathrm{I}^{2}=0 \%$; Figure 2), and fewer intraoperative blood loss (MD $=-80.53,95 \% \mathrm{Cl}$ of -104.75 to $-56.32, P<0.01, I^{2}=90 \%$; Figure 2) than the fusion group.

The test for heterogeneity of surgical time showed remarkable heterogeneity $\left(I^{2}=88 \%\right)$, so sensitivity analysis was performed. The influence of each study was evaluated by sequential removal of trials. The results showed that the studies of Fei et al. ${ }^{3}$, Yang et al. ${ }^{19}$, and Liu et al. ${ }^{28}$ led to high heterogeneity. We drew the same conclusion (MD $=-21.07,95 \% \mathrm{Cl}$ of -25.53 to $-16.62, P<0.01, \mathrm{I}^{2}=0 \%$ ) as before when we excluded these studies. The differences in skill of surgeons may cause this heterogeneity. Furthermore, sensitivity analysis was performed due to the remarkable heterogeneity of intraoperative blood loss $\left(I^{2}=\right.$ $90 \%)$. The final result (MD $=-135.98,95 \% \mathrm{Cl}$ of -163.56 to $-108.39, P<0.01, \mathrm{I}^{2}=0 \%$ ) was consistent with the abovementioned outcome.

\section{In-hospital complications}

Seven studies $3,4,7,16,19,24,29$ provided data on in-hospital complications. The pooled results indicated that the Dynesys group was associated with significantly fewer in-hospital complications than the fusion group $\left(\mathrm{RR}=0.49,95 \% \mathrm{Cl} 0.26-0.93, P=0.03 ;\left.\right|^{2}=0 \%\right.$; Figure 2 ).

\section{Clinical outcomes at final follow-up}

\section{Postoperative VAS scores}

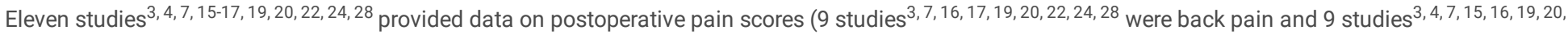
22,28 were leg pain) between Dynesys and fusion groups. The combined results indicated that the postoperative VAS scores for low back pain (MD $=-0.23$, $95 \% \mathrm{Cl}$ of -0.31 to $-0.16, P<0.01, \mathrm{I}^{2}=12 \%$; Figure 3 ) and leg pain (MD $=-0.30,95 \% \mathrm{Cl}$ of -0.41 to $-0.19, P<0.01, \mathrm{I}^{2}=38 \%$; Figure 3 ) in the DS group were better than those in the fusion group. The Egger's test suggested no publication biases of VAS scores for back (coefficient $=-2.17, \mathrm{SE}=3.44, P=0.548)$ and leg pain (coefficient $=-3.32, \mathrm{SE}=2.73, P=0.264$ ).

\section{Postoperative ODI scores}

Nine studies $3,4,7,16-18,22,24,28$ provided data on postoperative ODI scores. The combined results revealed that the Dynesys group was insignificantly different from the fusion group in terms of postoperative $\mathrm{ODI}$ scores (MD $=0.24,95 \% \mathrm{Cl}$ of -0.69 to $1.17, P=0.61, \mathrm{I}^{2}=0 \%$; Figure 3 ). The Egger's test suggested no publication bias (coefficient $=-1.24, \mathrm{SE}=1.33, P=0.382$ ).

\section{Screw loosening and breakage}

Eight studies $^{7,15-17,19,21,22,24}$ and four studies ${ }^{3,4,7,15}$ provided data on screw loosening and breakage, respectively. The pooled results showed that the Dynesys group was insignificantly different from the fusion group in terms of screw loosening ( $\mathrm{RR}=1.17,95 \% \mathrm{Cl} 0.70-1.96, P=0.55 ; \mathrm{I}^{2}=0 \%$; Figure 4$)$ and screw breakage $\left(\mathrm{RR}=0.89,95 \% \mathrm{Cl} 0.32-2.46, P=0.82 ; I^{2}=29 \%\right.$; Figure 4). The Egger's test suggested no publication bias of screw loosing (coefficient= $0.003, \mathrm{SE}=-0.70, P=0.996)$.

\section{Surgical reoperation}

Five studies 3 , 16, 17, 21, 24 provided data on surgical reoperation. The pooled results indicated that the Dynesys group was insignificantly different from the fusion group in terms of surgical reoperation ( $\mathrm{RR}=0.48,95 \% \mathrm{Cl} 0.19-1.18, P=0.11 ; \mathrm{I}^{2}=0 \%$; Figure 4$)$.

\section{Radiographic outcomes}

\section{Postoperative ROM}

Five studies $3,15,19,20,22$ provided data on ROM at stabilized segments. The pooled data indicated that the ROM at the stabilized segment in the fusion group decreased significantly than that in the Dynesys group ( $\mathrm{MD}=2.79,95 \% \mathrm{Cl}$ of 1.84 to $3.75, P<0.01, \mathrm{I}^{2}=97 \%$; Figure 5 ). Sensitivity analysis was performed by sequential removal of the included trials due to the remarkable heterogeneity $\left(I^{2}=97 \%\right)$. The results showed the same conclusion $(\mathrm{MD}=1.72,95 \% \mathrm{Cl}$ of 1.65 to $\left.1.78, P<0.01, I^{2}=0 \%\right)$ as before. 
Six studies $4,7,16,19,20,24$ provided data on ROM at proximal adjacent segment. The combined results indicated that the ROM in the fusion group increased significantly than that in the Dynesys group ( $M D=-1.88,95 \% \mathrm{Cl}$ of -2.85 to $-0.91, P<0.01, I^{2}=95 \%$; Figure 5). Sensitivity analysis was performed because of remarkable heterogeneity $\left(\mathrm{I}^{2}=95 \%\right)$. The result $\left(\mathrm{MD}=-2.62,95 \% \mathrm{Cl}\right.$ of -3.23 to $\left.-2.01, P<0.01, \mathrm{I}^{2}=0 \%\right)$ was consistent with the above outcome after removal of the two studies ${ }^{4,19}$ that caused heterogeneity.

Three studies $3,15,20$ provided data on the ROM of L1-S1 lumbar lordosis (LL) angle. The combined results indicated that the ROM in the fusion group decreased significantly than that in the Dynesys group ( $\mathrm{MD}=2.47,95 \% \mathrm{Cl}$ of 1.24 to $3.70, P<0.01, \mathrm{I}^{2}=36 \%$; Figure 5 ). After the sensitivity analysis, the final result $\left(\mathrm{MD}=2.08,95 \% \mathrm{Cl}\right.$ of 1.33 to $\left.2.83, P<0.01, \mathrm{I}^{2}=0 \%\right)$ confirmed the above outcome.

Postoperative disc heigh

Four studies ${ }^{7,16,19,24}$ reported the disc heigh at the intervertebral segment and three studies ${ }^{7,16,24}$ reported the disc heigh at the proximal adjacent segment. The pooled results showed that the Dynesys group was insignificantly different from the fusion group in terms of the disc heigh at the intervertebral segment $\left(\mathrm{MD}=-0.72,95 \% \mathrm{Cl}\right.$ of -1.58 to $0.15, P=0.11, \mathrm{I}^{2}=87 \%$; Figure 6$)$ and the disc heigh at the proximal adjacent segment $(\mathrm{MD}=-0.25,95 \% \mathrm{Cl}$ of -0.70 to 0.21 , $P=0.29,1^{2}=0 \%$; Figure 6).

\section{ASP}

ASDeg (Radiographic ASD)

Five studies ${ }^{15}, 16,22,24,29$ provided data on ASDeg. The pooled results indicated that the Dynesys group showed less ASDeg than the fusion group (RR $=0.50$, $95 \%$ Cl $0.35-0.73, P<0.01 ;\left.\right|^{2}=10 \%$; Figure 7).

ASDis (symptomatic ASD)

Three studies ${ }^{15,19,24}$ provided data on ASDis. The pooled results showed that the Dynesys group was insignificantly different from the fusion group in terms of ASDis (RR $=0.49,95 \% \mathrm{Cl} 0.11-2.08, P=0.33 ;\left.\right|^{2}=0 \%$; Figure 7 ).

\section{Discussion}

Spine fusion is a primary therapy for spinal degenerative diseases but is associated with several complications, especially acceleration of ASP. ${ }^{1,5,12}$ To prevent the complications caused by fusion method, scholars have developed dynamic stabilization techniques, including DS, which is one of the widely used non-fusion techniques and has the advantage of ASP prevention. ${ }^{3}$ However, inconsistent results have been reported regarding the advantages of DS over fusion method in terms of clinical outcomes. 3 , 5-7, 20-23 Whether DS can retain ROM at surgical segment and reduce ASP also remain controversial.

Furthermore, most of the published studies mainly focused on short-term clinical efficiency, whereas reports on medium-and long-term outcomes are rare and have conflicting findings, especially trials involving fusion method as control. $7,16,17,24,29$ Hence, we conducted a meta-analysis to compare the radiographic and clinical outcomes between DS and instrumented fusion in patients with or without grade I spondylolisthesis at a minimum follow-up period of 2 years.

Many studies have confirmed the safety and clinical equivalence of DS to fusion method. ${ }^{4,21,24,30}$ DS had been proved to relieve clinical symptoms and improve functional status as well as fusion instruments with durations of follow-up ranging from 28.78 to 70.14 months. ${ }^{7,16-18,20}$ Furthermore, Bredin et al. ${ }^{15}$ performed a study with a follow-up of 93.6 months and concluded that DS group showed significant improvements in VAS and ODI scores than fusion method. In this meta-analysis, we draw the conclusion that DS remarkably improved the VAS scores for back and leg pain. Meanwhile, no significant difference in postoperative ODI scores was detected between the two groups. We also found that DS was associated with shorter surgical time, fewer intraoperative blood loss, shorter length of hospital stay, and less in-hospital complications. The results may be explained as follows. Although the decompression of nerve roots was conducted in DS and fusion methods, the risk of nerve root injuries increased due to the fact that the latter not only dissects the bone and soft tissue but also requires the preparation of endplates and insertion of interbody devices or bone grafting, leading to back and leg pain after surgery. Meanwhile, additional surgical instrument and enlargement of surgical trauma in fusion method increased the surgical time, blood loss, and inhospital complications. Furthermore, the occurrence of non-fusion with fusion method would aggravate clinical symptoms in the later recovery process. Moreover, we demonstrated that the Dynesys group was insignificantly different from the fusion group in terms of screw loosening, screw breakage, and surgical revision. These results might be attributed to the simplified operation of DS and the preservation of lumbar mobility. Hence, we conclude that DS showed equivalent, or even better clinical measurements, compared with conventional fusion. The safety of DS has complied with the basic requirements for widespread clinical application in cases meeting indications such as disc herniation, lumbar spinal stenosis, and grade I degenerative spondylolisthesis in lumbar.

The major aim of utilizing DS is to minimize ASP. ${ }^{6,31}$ Hashimoto et al. ${ }^{8}$ reported that the fusion of stabilized segments may increase the biomechanical stress on the adjacent levels, leading to ASDeg and ASDis. Many studies have proven that DS preserved the ROM at the stabilized segments and prevented hypermobility at the adjacent segments in the medium- or long-term follow-up. ${ }^{4,7,16,20,21,24,30}$ However, Yang et al. ${ }^{19}$ drew an opposite conclusion that DS did not maintain the ROM at the adjacent segments compared with fusion method after a minimum follow-up of 24 months. Furthermore, Schaeren et al. ${ }^{32}$ found no measurable motion at the stabilized segments after DS but reported sign of degeneration at adjacent segments in $47 \%$ patients after 4 years; this finding is similar to that obtained using fusion instrument. Hence, results regarding maintenance on the ROM with DS are conflicting. In our meta-analysis, we reported that in the fusion group, the ROM at stabilized segments and the ROM of LL decreased remarkably, but the ROM at adjacent segments increased remarkably than those in the DS group. The result may be explained by the fact that the DS pedicle screws are connected to the PET cords, thereby providing tension to 
limit excessive flexion and PCU spacers resisting compressive force to limit over extension; this phenomenon leads to reduced vertebral abnormal activity and preserved motion at the instrumental segments and hypermobility at the adjacent segments in the medium- and long-term duration. ${ }^{33}$

Another early radiographic manifestation of ASP in clinical practice is the narrowness and loss of the intervertebral space, the height of which is commonly considered an indicator to evaluate the degree of ASP. ${ }^{3,16} \mathrm{Hu}$ et al. ${ }^{7}$ found that the disc heigh at the stabilized and adjacent segments decreased in the DS and fusion groups after a minimum follow-up of 5 years, while

no significant difference of the decrease was found between the two groups. Moreover, in the study conducted by Fei et al. ${ }^{3}$, the disc heigh at the stabilized segments in the DS group increased in a short-term period and then decreased in the long-term follow-up. With the progress of disc degeneration, the influence of instrumentation is more obvious after long-term follow-up. In the meta-analysis, we concluded that the Dynesys group was insignificantly different from the fusion group in terms of disc heigh both at stabilized and adjacent segments. The results can be attributed to the natural degenerative progression of the disc at the stabilized and adjacent segments despite DS, which is irrelative to the stabilization method. ${ }^{34,35}$ Thus, DS showed no advantage over fusion method in prevention of disc degeneration after surgery.

Whether DS can delay the occurrence of ASP continues to be controversial. Bredin et al. ${ }^{15}$ performed a study with a mean follow-up of 5.5 years and reported significantly less ASDeg in the DS group than in the fusion group (12.1\% vs. $36 \%)$; meanwhile, Zhang et al. ${ }^{24}$ confirmed the conclusion in their study with mean follow-up of 55.2 months. Scholars proposed that DS may not prevent ASDeg, with high rates ranging from $16 \%$ to $47 \%$. ${ }^{31,32,36}$ In the meta-analysis, we found that the Dynesys group showed less ASDeg than the fusion group. Although DS showed no superiority in preventing disc degeneration compared with fusion method, the former performed well in sparing abnormal biomechanical load at the stabilized and adjacent segments, consequently preserving the physiological motion after stabilization; this finding may explain why DS can prevent ASDeg. ${ }^{10,37}$ Furthermore, the PET cords and PCU spacers were utilized to restrict flexion and extension, respectively. With increasing cord pretension, the flexion ROM at stabilized and adjacent levels increased, but the extension ROM decreased. ${ }^{38}$ The realistic stiffness of DS varies with cord pretension and spacer length, which are decided based on surgeons' personal experience; this phenomenon can partly explain the conflicting results in previously published works. ${ }^{7,39,40}$

The prevention of ASDis is crucial due to the potential risk of revision surgery and adverse impact on health care outcomes and cost. Some scholars reported no significant difference in ASDis between the two groups with a minimum follow-up of 53.6 months. ${ }^{15,24}$ However, St-Pierre et al. ${ }^{23}$ reported that DS was associated with a high rate of ASDis (29\%) after a mean follow-up of 92 months. Our meta-analysis demonstrated that the Dynesys group showed less ASDis than the fusion group, whereas the difference was not significant. The result revealed that DS can prevent ASDeg instead of ASDis compared with fusion method. ASDeg represents radiographic etiologies, while ASDis represents clinical symptoms corelated with one of the radiographic degenerations. The occurrence of ASDis is relatively lower than that of ASDeg because not all ASDeg will cause clinical symptoms. Furthermore, the lack of remarkable difference in disabilities (ODI) postoperatively between the two methods interpreted the similar incidence of ASDis after surgery.

This meta-analysis has limitations. First, only one RCT along with 13 comparative cohort studies was included, resulting in less powerful results compared with that obtained purely from RCTs. Second, the definitions of ASDed and ASDis encountered in previous studies were varied and ambiguous. No consensus criterion has been proposed to define ASDed and ASDis, leading to imprecise outcome measures. Third, the number of the included studies was limited in terms of the length of hospital stay, ROM of LL, disc heigh at proximal adjacent segments, and ASDis. Future work should employ more RCTs of high quality and long-term follow-up trials within different cultural contexts.

\section{Conclusion}

DS showed equivalent outcomes in terms of ODI, screw loosing, screw breakage, and surgical revision compared with fusion method; moreover, DS had better clinical measurements in terms of surgical time, intraoperative blood loss, length of hospital stay, VAS, and in-hospital complications than fusion instrument. Furthermore, DS provided additional benefits in preserving the motion at the stabilized segments, limiting the hypermobility at the adjacent segments, and consequently preventing ASDeg compared with fusion method. Dynesys stabilization represented a complementary choice in the treatment of degenerative spinal lumbar disease with or without grade I spondylolisthesis.

\section{Abbreviations}

DS: Dynesys stabilization; ROM: range of motion; DH: disc height; ASDeg: adjacent segment degeneration; ASDis: adjacent segment disease; ASP: adjacent segment pathology; PRISMA: the Preferred Reporting Items for Systematic Reviews and Meta-analyses; VAS: visual analogue scale; ODI: Oswestry disability index; RCTs: randomized controlled trials; RR: risk ratio; SMD: standardized mean difference

\section{Declarations}

\section{Disclosures}

Conflict of interest The authors declare that they have no conflict of interest concerning the materials or methods used in this study or the findings specified in this paper.

\section{Competing interests}

All authors confirmed that there is no conflict of interest regarding the submitted manuscript. 
Funding

This trial is not founded by any funding.

\section{Availability of data and materials}

All data generated or analyzed during this study are included in this published article and its supplementary information files.

\section{Ethics approval and consent to participate}

This trial is a meta-analysis, which we collected data from other included studies. Ethics approval and consent to participate is not applicable.

\section{Consent for publication}

This trial is a meta-analysis, which we collected data from other included studies. Consent for publication is not applicable.

\section{References}

1. Yavin D, Casha S, Wiebe S, Feasby TE, Clark C, Isaacs A, Holroyd-Leduc J, Hurlbert RJ, Quan H, Nataraj A et al: Lumbar fusion for degenerative disease: A systematic review and meta-analysis. Neurosurgery 2017, 80(5):701-715.

2. Lee JC, Choi S-W: Adjacent segment pathology after lumbar spinal fusion. Asian Spine J 2015, 9(5):807-817.

3. Fei H, Xu J, Wang S, Xie Y, Ji F, Xu Y: Comparison between posterior dynamic stabilization and posterior lumbar interbody fusion in the treatment of degenerative disc disease: a prospective cohort study. J Orthop Surg Res 2015, 10:87.

4. Wang Q, Liu J, Shi Y, Chen Y, Yu H, Ma J, Ren W, Yang H, Wang H, Xiang L: Short-term effects of a dynamic neutralization system (Dynesys) for multisegmental lumbar disc herniation. Eur Spine J 2016, 25(5):1409-1416.

5. Zhang Y, Zhang ZC, Li F, Sun TS, Shan JL, Guan K, Zhao GM, Zhang LZ: Long-term outcome of dynesys dynamic stabilization for lumbar spinal stenosis. Chin Med J (Engl) 2018, 131(21):2537-2543.

6. Zhang C, Berven SH, Fortin M, Weber MH: Adjacent Segment Degeneration Versus Disease After Lumbar Spine Fusion for Degenerative Pathology. Clin Spine Surg 2016, 29(1):21-29.

7. Hu A, Sun C, Liang Y, Wang H, Li X, Dong J: Multi-segmental lumbar spinal stenosis treated with Dynesys stabilization versus lumbar fusion in elderly patients: a retrospective study with a minimum of 5 years' follow-up. Arch Orthop Trauma Surg 2019, 139(10):1361-1368.

8. Hashimoto K, Aizawa T, Kanno H, Itoi E: Adjacent segment degeneration after fusion spinal surgery-a systematic review. Int Orthop 2019, 43(4):987-993.

9. Huang Y-J, Zhao S-J, Zhang Q, Nong L-M, Xu N-W: Comparison of lumbar pedicular dynamic stabilisation systems versus fusion for the treatment of lumbar degenerative disc disease: a meta-analysis. Acta Orthop Belg 2017, 83(1):180-193.

10. Lee SE, Jahng T-A, Kim H-J: Clinical experiences of non-fusion dynamic stabilization surgery for adjacent segmental pathology after lumbar fusion. Int $J$ Spine Surg 2016, 10:8.

11. Fayyazi AH, Ordway NR, Park S-A, Fredrickson BE, Yonemura K, Yuan HA: Radiostereometric analysis of postoperative motion after application of Dynesys dynamic posterior stabilization system for treatment of degenerative spondylolisthesis. J Spinal Disord Tech 2010, $23(4): 236-241$.

12. Akyoldas G, Cevik OM, Suzer T, Sasani M, Oktenoglu T, Ozer AF: Dynamic Stabilization of the Lumbar Spine Using the Dynesys System. Turk Neurosurg 2020, 30(2):190-193.

13. Pham MH, Mehta VA, Patel NN, Jakoi AM, Hsieh PC, Liu JC, Wang JC, Acosta FL: Complications associated with the Dynesys dynamic stabilization system: a comprehensive review of the literature. Neurosurg Focus 2016, 40(1):E2.

14. Prud'homme M, Barrios C, Rouch P, Charles YP, Steib J-P, Skalli W: clinical outcomes and complications after pedicle-anchored dynamic or hybrid lumbar spine stabilization: a systematic literature review. J Spinal Disord Tech 2015, 28(8):E439-E448.

15. Bredin S, Demay O, Mensa C, Madi K, Ohl X: Posterolateral fusion versus Dynesys dynamic stabilization: retrospective study at a minimum 5.5 years' follow-up. Orthop Traumatol Surg Res 2017, 103(8):1241-1244.

16. Wu H, Pang Q, Jiang G: Medium-term effects of Dynesys dynamic stabilization versus posterior lumbar interbody fusion for treatment of multisegmental lumbar degenerative disease. J Int Med Res 2017, 45(5):1562-1573.

17. Haddad B, Makki D, Konan S, Park D, Khan W, Okafor B: Dynesys dynamic stabilization: less good outcome than lumbar fusion at 4-year follow-up. Acta Orthop Belg 2013, 79(1):97-103.

18. He J, Li J, Luo C, Sun Y, Nong L, Xie H: Impact of the Dynesys dynamic stabilization system on the fixation-adjacent intervertebral discs. Int J Clin Exp Med 2016, 9(7):14698-14703.

19. Yang M, Li C, Chen Z, Bai Y, Li M: Short term outcome of posterior dynamic stabilization system in degenerative lumbar diseases. Indian J Orthop 2014, 48(6):574-581.

20. Zhang C, Wang L, Hou T, Luo L, Zhao C, Gan Y, Zhou Q, Li P: The influence of L4-S1 Dynesys ${ }^{\circledR}$ dynamic stabilization versus fusion on lumbar motion and its relationship with lumbar degeneration: a retrospective study. J Orthop Surg Res 2017, 12(1):99.

21. Yu S-W, Yang S-C, Ma C-H, Wu C-H, Yen C-Y, Tu Y-K: Comparison of Dynesys posterior stabilization and posterior lumbar interbody fusion for spinal stenosis L4L5. Acta Orthop Belg 2012, 78(2):230-239. 
22. Kuo C-H, Huang W-C, Wu J-C, Tu T-H, Fay L-Y, Wu C-L, Cheng H: Radiological adjacent-segment degeneration in L4-5 spondylolisthesis: comparison between dynamic stabilization and minimally invasive transforaminal lumbar interbody fusion. J Neurosurg Spine 2018, 29(3):250-258.

23. St-Pierre GH, Jack A, Siddiqui MMA, Henderson RL, Nataraj A: Nonfusion does not prevent adjacent segment disease: Dynesys long-term outcomes with minimum five-year follow-up. Spine (Phila Pa 1976) 2016, 41(3):265-273.

24. Zhang Y, Shan J-L, Liu X-M, Li F, Guan K, Sun T-S: Comparison of the dynesys dynamic stabilization system and posterior lumbar interbody fusion for lumbar degenerative disease. PLoS One 2016, 11(1):E0148071.

25. Liberati A, Altman DG, Tetzlaff J, Mulrow C, Gøtzsche PC, loannidis JPA, Clarke M, Devereaux PJ, Kleijnen J, Moher D: The PRISMA statement for reporting systematic reviews and meta-analyses of studies that evaluate health care interventions: explanation and elaboration. PLoS Med 2009, 6(7): E1000100.

26. Higgins JPT, Green S: Cochrane handbook for systematic reviews of interventions version 5.1.0. The Cochrane Collaboration, 2011. http://www.cochranehandbook.org. Accessed March 20, 2012.

27. Wells GA, Shea B, O'Connell D, Robertson J, Peterson J, Welch V, Losos M, Tugwell P: The Newcastle-Ottawa Scale (NOS) for assessing the quality of nonrandomized studies in meta-analysis. 2011. http://www.ohri.ca/programs/clinical epidemiology/oxford.asp Accessed April 15, 2012.

28. Liu K, Sun W, Lu Q, Chen J, Tang J: A cost-utility analysis of Dynesys dynamic stabilization versus instrumented fusion for the treatment of degenerative lumbar spine diseases. J Orthop Sci 2017, 22(6):982-987.

29. Silvestre MD, Lolli F, Bakaloudis G: Degenerative lumbar scoliosis in elderly patients: dynamic stabilization without fusion versus posterior instrumented fusion. Spine J 2014, 14(1):1-10.

30. Veresciagina K, Mehrkens A, Schären S, Jeanneret B: Minimum ten-year follow-up of spinal stenosis with degenerative spondylolisthesis treated with decompression and dynamic stabilization. J Spine Surg 2018, 4(1):93-101.

31. Hoppe S, Schwarzenbach O, Aghayev E, Bonel H, Berlemann U: Long-term outcome after monosegmental L4/5 stabilization for degenerative spondylolisthesis with the Dynesys device. Clin Spine Surg 2012, 29(2):72-77.

32. Schaeren S, Broger I, Jeanneret B: Minimum four-year follow-up of spinal stenosis with degenerative spondylolisthesis treated with decompression and dynamic stabilization. Spine (Phila Pa 1976) 2008, 33(18):E636-E642.

33. Más Y, Gracia L, Ibarz E, Gabarre S, Peña D, Herrera A: Finite element simulation and clinical follow-up of lumbar spine biomechanics with dynamic fixations. PLoS One 2017, 12(11):E0188328.

34. Kumar A, Beastall J, Hughes J, Karadimas EJ, Nicol M, Smith F, Wardlaw D: Disc changes in the bridged and adjacent segments after Dynesys dynamic stabilization system after two years. Spine (Phila Pa 1976) 2008, 33(26):2909-2914.

35. Klöckner C: Long-term results of the Dynesys implant. Orthopade 2010, 39(6):559-564.

36. Hoff E, Strube P, Gross C, Putzier M: Sequestrectomy with additional transpedicular dynamic stabilization for the treatment of lumbar disc herniation: No clinical benefit after 10 years follow-up. Spine (Phila Pa 1976) 2013, 38(11):887-895.

37. Shih S-L, Liu C-L, Huang L-Y, Huang C-H, Chen C-S: Effects of cord pretension and stiffness of the dynesys system spacer on the biomechanics of spinal decompression- a finite element study. BMC Musculoskelet Disord 2013, 14:191.

38. Liu C-L, Zhong Z-C, Hsu H-W, Shih S-L, Wang S-T, Hung C, Chen C-S: Effect of the cord pretension of the dynesys dynamic stabilisation system on the biomechanics of the lumbar spine: A finite element analysis. Eur Spine $J$ 2011, 20:1850-1858.

39. Lee C-H, Jahng T-A, Hyun S-J, Kim CH, Park S-B, Kim K-J, Chung CK, Kim H-J, Lee S-E: Dynamic stabilization using the Dynesys system versus posterior lumbar interbody fusion for the treatment of degenerative lumbar spinal disease: a clinical and radiological outcomes-based meta-analysis.

Neurosurgical focus 2016, 40(1):E7.

40. Jahng T-A, Kim YE, Moon KY: Comparison of the biomechanical effect of pedicle-based dynamic stabilization: A study using finite element analysis. Spine J 2013, 13(1):85-94.

\section{Tables}

Table 1. Characteristics of included studies 


\begin{tabular}{|c|c|c|c|c|c|c|c|c|c|c|c|c|}
\hline \multirow[t]{2}{*}{ Study } & \multirow{2}{*}{$\begin{array}{l}\text { Study } \\
\text { design }\end{array}$} & \multirow{2}{*}{ Country } & \multirow{2}{*}{$\begin{array}{l}\text { Study } \\
\text { Period }\end{array}$} & \multicolumn{2}{|l|}{ Age (years) } & \multicolumn{2}{|l|}{$\operatorname{Sex}(M / F)$} & \multicolumn{2}{|l|}{$\begin{array}{l}\text { No. of } \\
\text { patients }\end{array}$} & \multirow{2}{*}{$\begin{array}{l}\text { No. of } \\
\text { treated } \\
\text { segments }\end{array}$} & \multirow{2}{*}{$\begin{array}{l}\text { Type } \\
\text { of } \\
\text { fusion }\end{array}$} & Follow-up(mor \\
\hline & & & & Dynesys & Fusion & Dynesys & Fusion & Dynesys & Fusion & & & Dynesys Fu \\
\hline $\begin{array}{l}\text { Hu et } \\
\text { al., } 2019\end{array}$ & Retro & Germany & $\begin{array}{l}2011- \\
2013\end{array}$ & $69.27 \pm 4.92$ & $\begin{array}{l}69.41 \pm \\
4.23\end{array}$ & $11 / 11$ & $22 / 22$ & 22 & 44 & $1-3$ & TLIF & $68.50 \pm 6.40$ \\
\hline $\begin{array}{l}\text { Kuo et } \\
\text { al., } 2018\end{array}$ & Retro & China & $\begin{array}{l}2007 \\
-2014\end{array}$ & $60.1 \pm 10.8$ & $58.0 \pm 11.7$ & $21 / 35$ & $8 / 15$ & 56 & 23 & 1 & TLIF & $35.2(24-89)$ \\
\hline $\begin{array}{l}\text { Zhang } \\
\text { et al., } \\
2017\end{array}$ & Retro & China & $\begin{array}{l}2010- \\
2014\end{array}$ & $48.28 \pm .44$ & $\begin{array}{l}50.10 \pm \\
1.77\end{array}$ & $18 / 9$ & $17 / 22$ & 27 & 29 & 2 & TLIF & $28.78 \pm 0.61$ \\
\hline $\begin{array}{l}\text { Wu et } \\
\text { al., } 2017\end{array}$ & Pro & China & $\begin{array}{l}2008- \\
2010\end{array}$ & $49.6 \pm 8.3$ & $52.5 \pm 6.9$ & $14 / 12$ & $18 / 22$ & 26 & 31 & $2-4$ & PLIF & $50.3(46-65)$ \\
\hline $\begin{array}{l}\text { Liu et } \\
\text { al., } 2017\end{array}$ & Pro & China & $\begin{array}{l}2011- \\
2013\end{array}$ & $52.9 \pm 10.3$ & $54.6 \pm 9.9$ & $26 / 29$ & $29 / 29$ & 55 & 58 & 1 & $\begin{array}{l}\text { PLIF } \\
+ \text { TLIF }\end{array}$ & At least 24 \\
\hline $\begin{array}{l}\text { Bredin } \\
\text { et al., } \\
2017\end{array}$ & Retro & France & $\begin{array}{l}2005- \\
2011\end{array}$ & $60.3 \pm 9.4$ & $61.3 \pm 8.4$ & $18 / 15$ & $13 / 12$ & 33 & 25 & $1-2$ & PLF & $93.6 \pm 16.5$ \\
\hline $\begin{array}{l}\text { Zhang } \\
\text { et al., } \\
2016\end{array}$ & Retro & China & $\begin{array}{l}2008- \\
2011\end{array}$ & $48.1 \pm 12.3$ & $52.3 \pm 12.9$ & $31 / 15$ & $37 / 13$ & 46 & 50 & $1-3$ & PLIF & $53.6 \pm 5.3$ \\
\hline $\begin{array}{l}\text { He et al., } \\
2016\end{array}$ & Pro & China & NA & $58.3 \pm 13.5$ & $61.4 \pm 15.2$ & $7 / 5$ & $9 / 15$ & 12 & 24 & $1-2$ & PLIF & $28.7 \pm 5.3$ \\
\hline $\begin{array}{l}\text { Wang et } \\
\text { al., } 2016\end{array}$ & Retro & China & $\begin{array}{l}2009- \\
2013\end{array}$ & $42.8 \pm 6.7$ & $56.8 \pm 6.2$ & $24 / 21$ & $17 / 23$ & 45 & 40 & $2-3$ & PLIF & $30.6 \pm 8.6$ \\
\hline $\begin{array}{l}\text { Fei et } \\
\text { al., } 2015\end{array}$ & Pro & China & $\begin{array}{l}2007- \\
2009\end{array}$ & $47.3 \pm 12.9$ & $52.9 \pm 11.2$ & $51 / 44$ & $40 / 41$ & 95 & 81 & $1-3$ & PLIF & $36-66$ \\
\hline $\begin{array}{l}\text { Yang et } \\
\text { al., } 2014\end{array}$ & Pro & China & $\begin{array}{l}2010- \\
2012\end{array}$ & $55.96 \pm 7.68$ & $54.69 \pm 3.26$ & $17 / 13$ & $21 / 24$ & 30 & 45 & $1-2$ & PLIF & $26.64 \pm 5.16$ \\
\hline $\begin{array}{l}\text { Silvestre } \\
\text { et al., } \\
2014\end{array}$ & Retro & Italy & $\begin{array}{l}2002- \\
2005\end{array}$ & $\begin{array}{l}68.4 \llbracket 61- \\
78 \rrbracket\end{array}$ & $\begin{array}{l}67.6 \rrbracket 62- \\
77 \rrbracket\end{array}$ & $7 / 25$ & $5 / 20$ & 32 & 25 & NA & PLIF & $64 \rrbracket 42-90 \rrbracket$ \\
\hline $\begin{array}{l}\text { Haddad } \\
\text { et al., } \\
2013\end{array}$ & Retro & UK & $\begin{array}{l}2004- \\
2006\end{array}$ & $40.6 \pm 6.46$ & $46.5 \pm 10.7$ & 19/13 & $15 / 17$ & 32 & 32 & NA & PLIF & 48 \\
\hline $\begin{array}{l}\text { Yu et al., } \\
2012\end{array}$ & RCT & China & $\begin{array}{l}2006- \\
2007\end{array}$ & $52.22 \pm 8.31$ & $\begin{array}{l}55.52 \pm \\
6.98\end{array}$ & $10 / 17$ & $11 / 18$ & 27 & 26 & 1 & PLIF & 36 \\
\hline
\end{tabular}

Abbreviations: RCT= randomized controlled trial; Pro= prospective clinical trial; Retro= retrospective clinical trial;

NOS= Newcastle-Ottawa Scale; TLIF= transforaminal lumbar interbody fusion; PLIF= posterior lumbar interbody fusion; PLF= posterolateral fusion.

$N A=$ not applicable

\section{Figures}




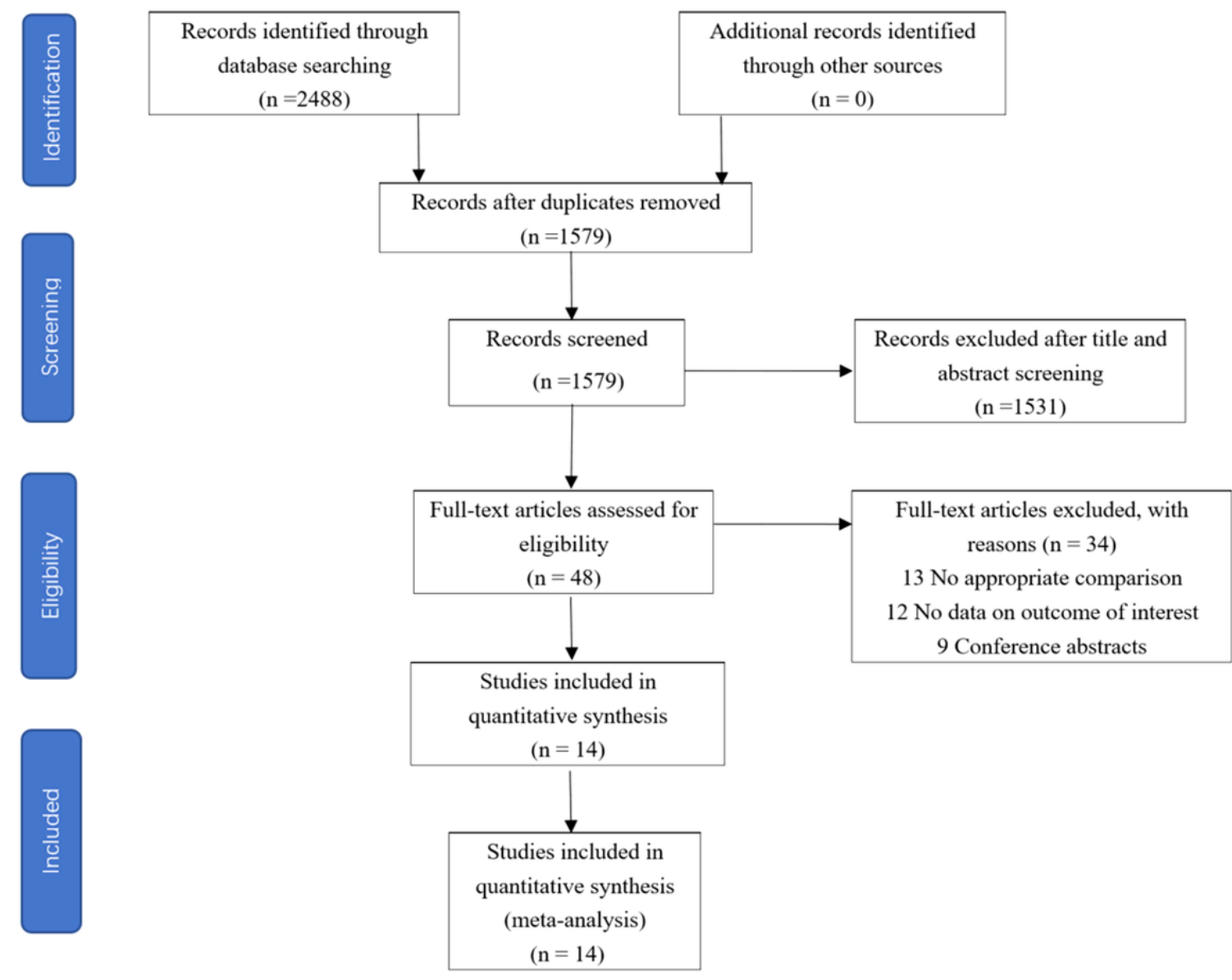

Figure 1. Flow diagram of study selection

\section{Figure 1}

Flow diagram of the study selection 


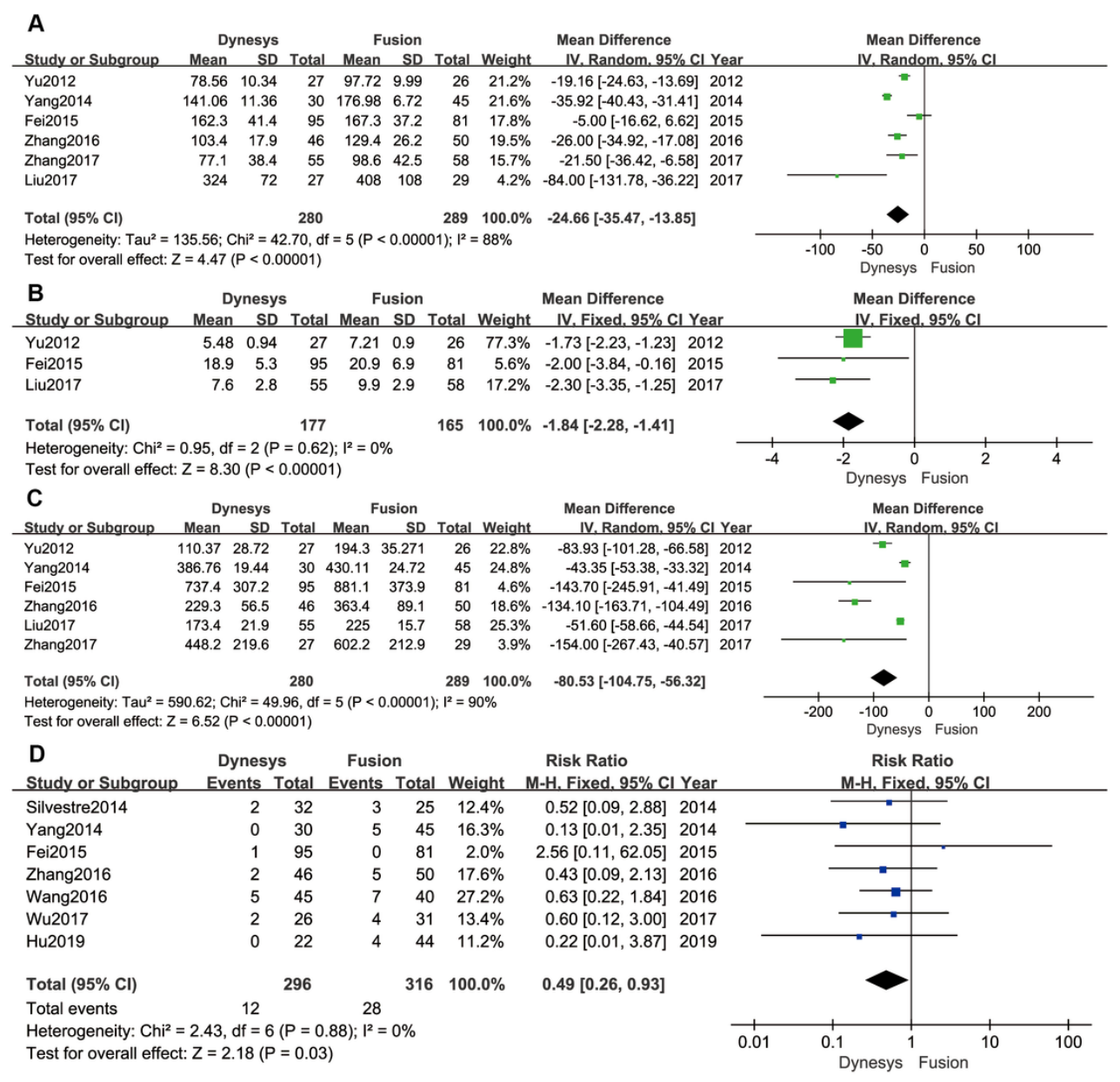

\section{Figure 2}

Forest plots of Dynesys stabilization versus instrumented fusion: (A) surgical time; (B) length of hospital stay; (C) intraoperative blood loss; (D) in-hospital complications. 


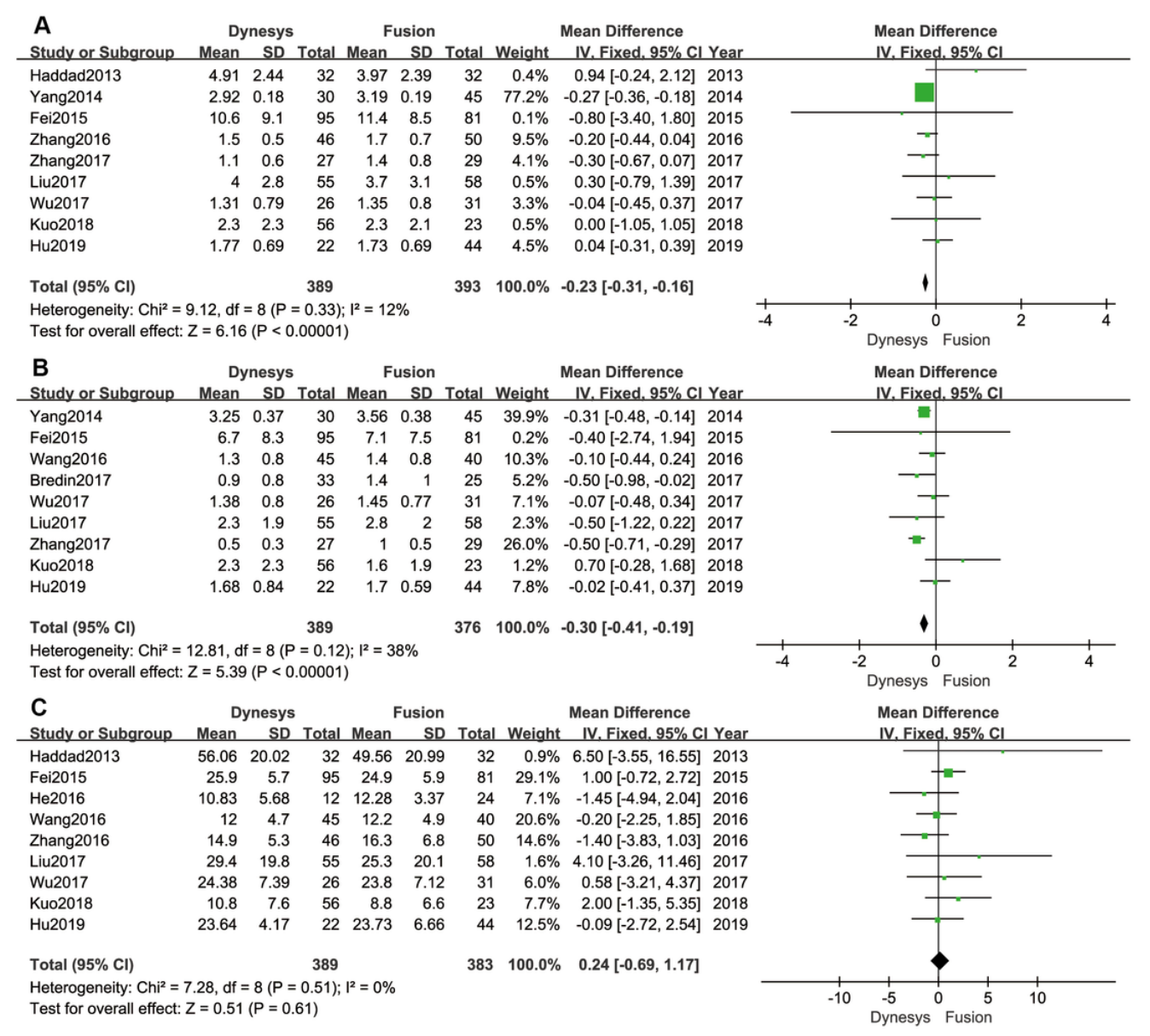

\section{Figure 3}

Forest plots of Dynesys stabilization versus instrumented fusion: (A) postoperative back VAS scores; (B) postoperative leg VAS scores; (C) postoperative ODI scores. 


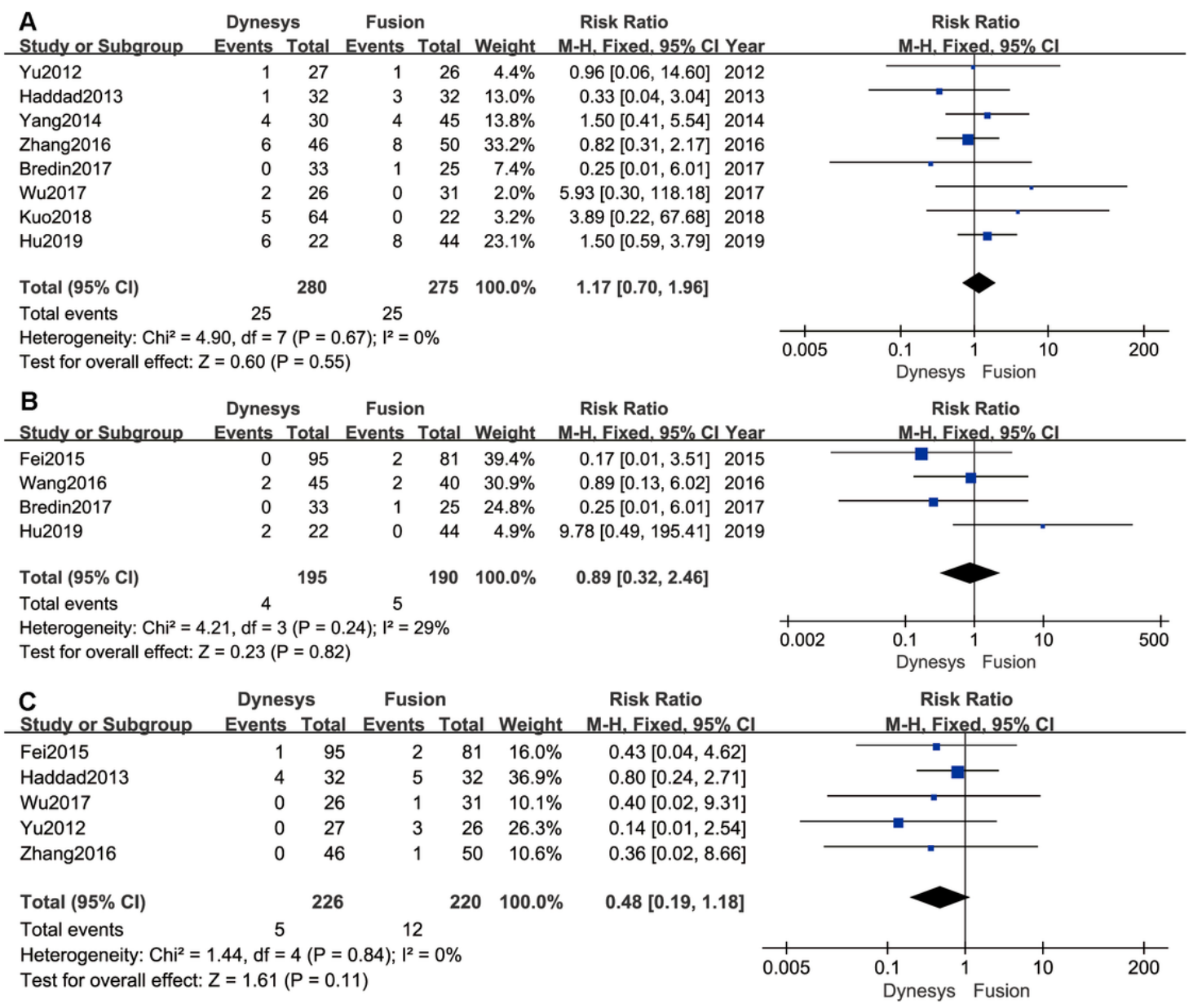

\section{Figure 4}

Forest plots of Dynesys stabilization versus instrumented fusion: (A) screw loosening; (B) screw breakage; (C) surgical reoperation. 


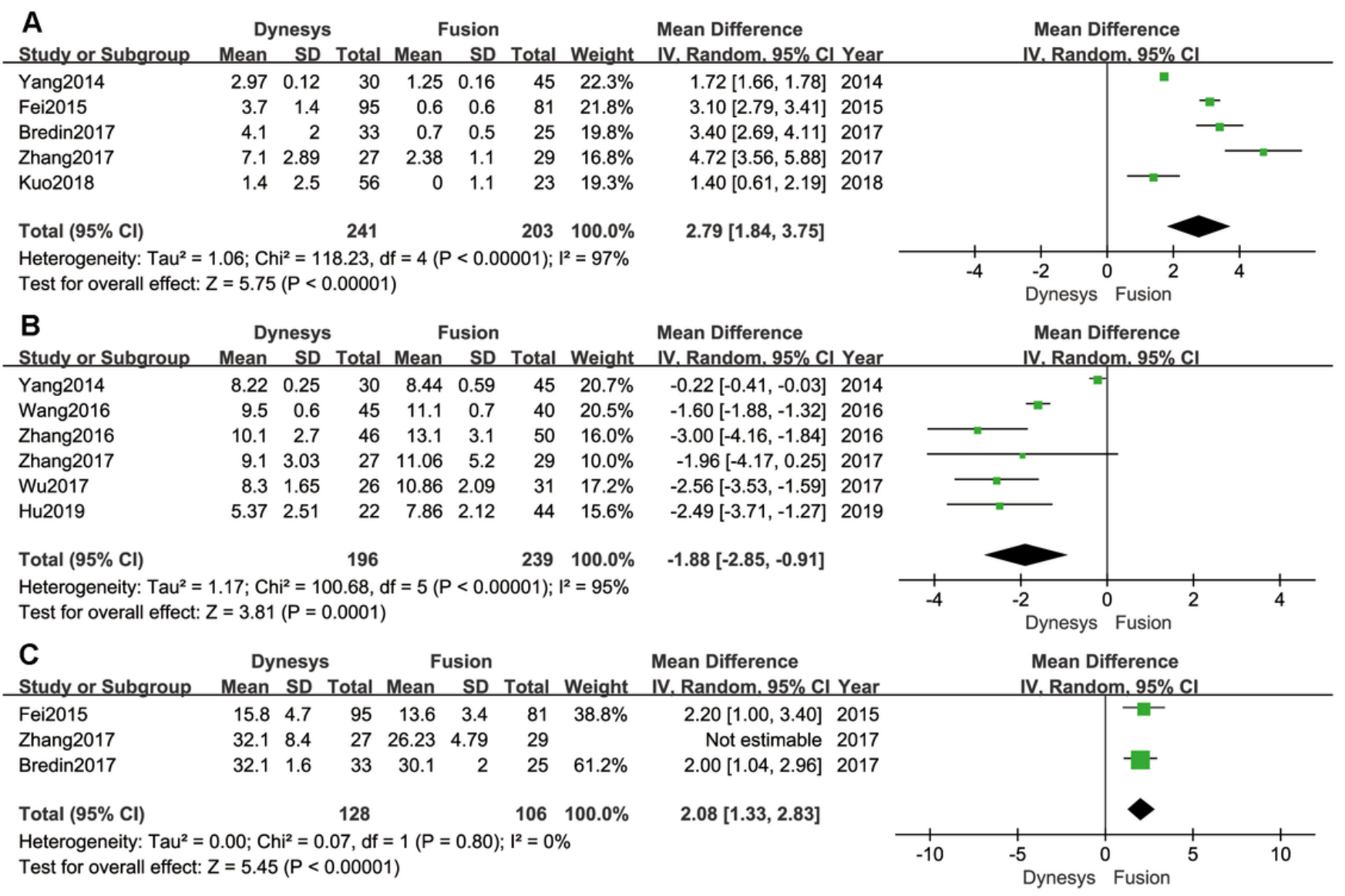

Figure 5

Forest plots of Dynesys stabilization versus instrumented fusion: (A) ROM at stabilized segments; (B) ROM at proximal adjacent segment; (C) ROM of L1-S1 lumbar lordosis angle.

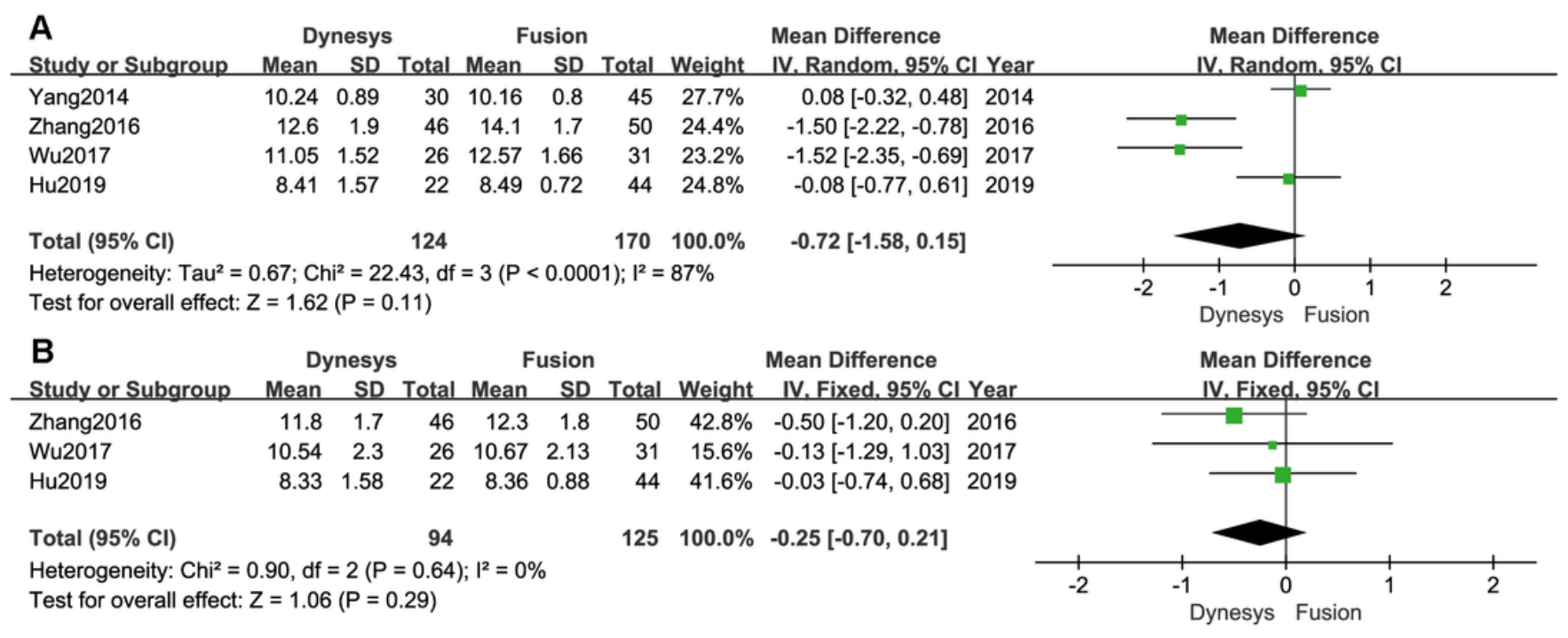


Figure 6

Forest plots of Dynesys stabilization versus instrumented fusion: (A) DH at the intervertebral segment; (B) DH at the proximal adjacent segment.

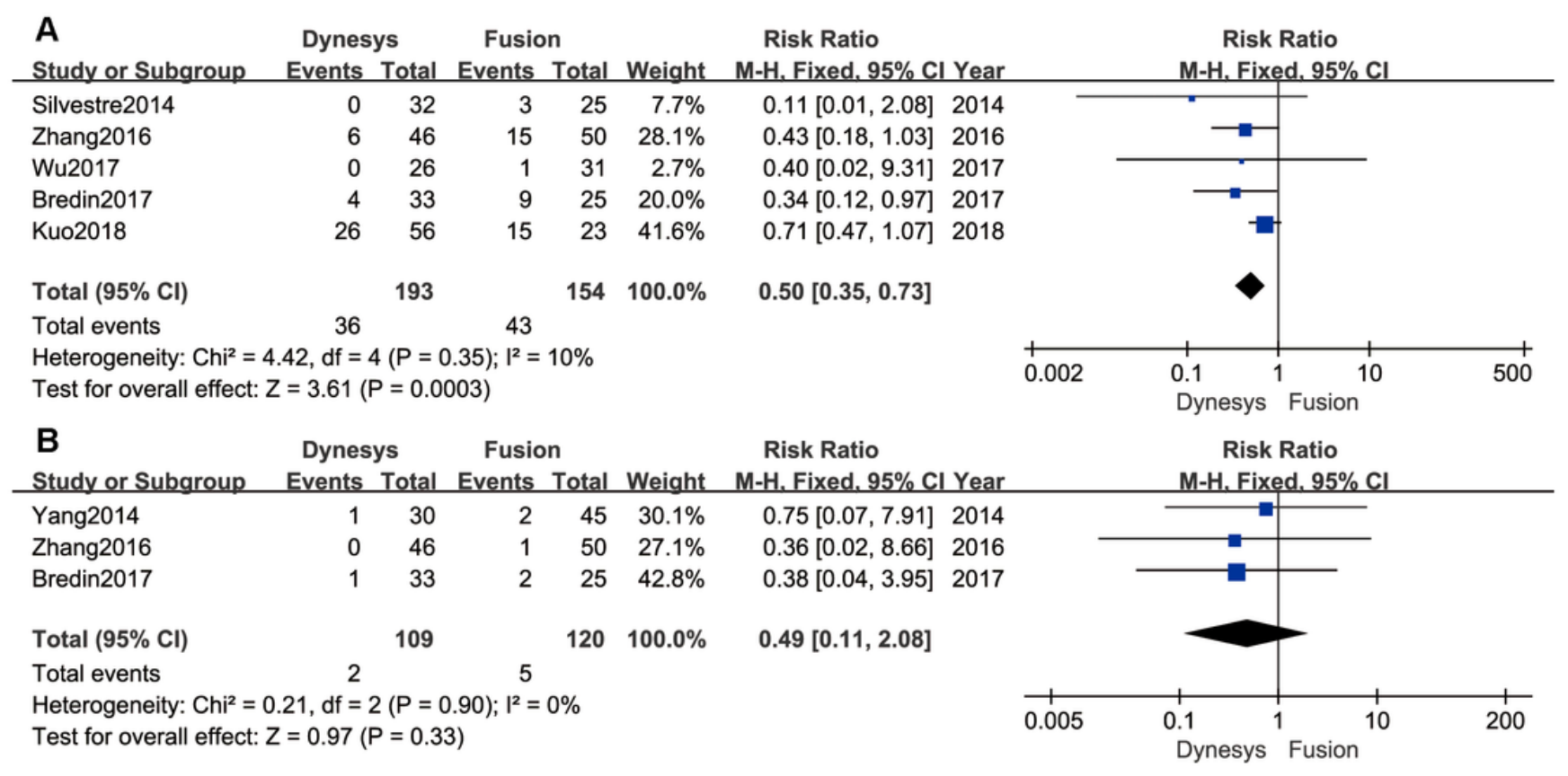

Figure 7

Forest plots of Dynesys stabilization versus instrumented fusion: (A) ASDeg; (B) ASDis.

\section{Supplementary Files}

This is a list of supplementary files associated with this preprint. Click to download.

- Additionalfile1.docx

- Additionalfile2.docx

- Additionalfile3.docx

- PRISMA2009checklist.doc 